Modeling value of time for trip chains using sigmoid utility

Peer-reviewed author version

USMAN, Muhammad; KNAPEN, Luk; YASAR, Ansar; BELLEMANS, Tom;

JANSSENS, Davy \& WETS, Geert (2017) Modeling value of time for trip chains

using sigmoid utility. In: Personal and Ubiquitous Computing, 21(6), p. 1041-1053.

DOI: $10.1007 / \mathrm{s} 00779-017-1030-4$

Handle: http://hdl.handle.net/1942/24089 


\title{
Modeling value of time for trip chains using sigmoid utility
}

\author{
Muhammad Usman*, Luk Knapen, Ansar-Ul-Haque Yasar, Tom Bellemans, Davy Janssens, Geert Wets \\ Transportation Research Institute (IMOB),University Hasselt, Diepenbeek, Belgium
}

\begin{abstract}
The use of modeling and simulation aids in deriving many decisions related to transportation planning and traffic operations. Representing the real systems via simulation allows exploring system behavior in an articulated way, which is often impossible in the real world. In this paper, a simulation-based framework is presented to evaluate the impact of congestion charging on daily activity plans of the individuals. Personal decision to accept the congestion charges is evaluated by comparing the value of time with congestion charge. Value of time varies throughout the day depending upon the time pressure at any moment exerted by preceding and succeeding activities. Time pressure during an activity increases if available time for that activity is insufficient to attain the perceived utility. Daily activities in the schedules are modeled using bell-shaped marginal utility that results in sigmoid utility. A model is presented which derives the activity specific parameters of the marginal utility function for the specific individual. To examine value of time of each person, the congestion charging is applied where personal willingness-to-pay is determined by comparing the ratios of cost to utility for original and adapted schedules. A large-scaled microsimulation of the modeled framework is used to simulate the whole population, which is created by FEATHERS, an operational activity-based model for Flanders, Belgium. The results of the simulation show that the number of individuals who avoid the congestion charges by adapting their schedules is almost three times the number of those who agree to pay it. The proposed framework can be useful to evaluate the tradeoff between value of time and costs where flexibility in selection of time defines the variability in cost.
\end{abstract}

Keywords: value of time, Sigmoid Utility, willingness-to-pay, Rescheduling, Travel behavior

\section{Introduction}

Representing the real systems via simulation allows exploring system behavior in an articulated way that is often either not possible, or too risky in the real world. The use of modeling and simulation within the engineering field is important and well recognized. It belongs to the tool set of all application areas and has been included in the body of knowledge. It has been applied to a broad range of topics in transportation sciences including simulation of vehicles or pedestrian flow, car following and lane changing models, route choice modeling, and traffic simulation. This is the key ingredient that drives most decisions in transportation planning and traffic operations.

Value-of-time determines the duration of the activity, switching to the next activity and willingness-to-pay travel cost for that activity. It varies throughout the day depending upon the time pressure at any moment exerted by preceding and succeeding activities. Time pressure during an activity increases if available time for that activity is insufficient to attain the envisaged utility. The difference in activity participation due to the unwanted situations may result in disproportion of time pressure. To balance the time pressure, the duration of all activities in the schedule is re-optimized to compensate the lost/gained time. A schedule is optimized by maximizing the satisfaction of individuals by activity participation. The maximum satisfaction can only be earned if all activities are started and finished at personal preferred time. Hence, maximum utilization of the time is bound by the preferred start time and duration of the activities that are planned for any specific day. In case of any time loss or deliberated shift in trip start time, traveler would re-optimize the timing of all activities in the schedule to achieve the maximum satisfaction using the available time resources.

To calculate the value of time, the researchers mostly use discrete choice modeling to select between alternatives for transport mode having different travel time and cost. Regression modeling is used to estimate the coefficients of cost

\footnotetext{
* Corresponding author. Tel.: +32-11-269 140; fax: +32-11- 269199 .

E-mail address: muhammad.usman@uhasselt.be
} 
and time to calculate the value of time. Value of time calculated using this method is always trip based and does not take into account other importance factors like time pressure introduced by internal deadlines or public transport availability during back home trip. For example, having three options of transport modes (bike, car and public transport), a traveler selects to travel by car for work trip because he has to pick someone while travelling back from work. In this research, the duration of all activities in the schedule is optimized to fulfill the constraints of maximization of time utilization and minimization of the total travel cost. To model the utility maximization constraint, the marginal utility for all activities is assumed to be bell shaped that result in sigmoid utility. Purpose of the research is to evaluate the proposed model for use in microsimulations. In case schedules need to be adapted in microsimulations, a behaviorally sound model is required to redistribute the lost/gained time in the activities that still are to be executed. We do not consider crisis or emergency situations in which behavior becomes very specific and different from regular situations. Our research covers (1) situations where individuals are forced to reschedule due to some unexpected events and (2) situations where individuals deliberately consider rescheduling because the environment conditions for the future change (e.g. congestion cost). The definition of VOT is cumbersome. Most researches consider a single trip and activity pair to determine a VOT value, which consequently, only holds for a specific situation (e.g. morning "home to work" trip or effect of evening trip in "morning mode choice"). In the context of congestion charging, individuals can influence the travel cost by adapting the timing in the schedule. In order to avoid the need for an absolute the VOT, we consider the ratio of $\Delta \mathrm{C} / \Delta \mathrm{U}$ change in cost verses change in utility. All car travelers who execute their trips during the specified period are charged the congestion cost. To avoid the congestion charges, car travelers intend to shift their overlapped trip and re-optimize their schedule by fixing the shifted trip. To examine value of time of each person, the congestion charging is applied where personal willingness-to-pay is determined by comparing the ratios of cost to utility for original and adapted schedules. A large-scaled microsimulation of the modeled framework is used to simulate the whole population, which is created by FEATHERS, an operational activity-based model for Flanders, Belgium. This paper is organized as follow: Section 2 provides the conceptual overview of the paper. Section 3.1 describes the theoretical model of the model that covers marginal utility and utility function and a method to derive the involved parameters. Section 3.2 and 3.3 provide the description of travel related costs and a method for schedule adaptation to avoid extra costs respectively. Section 3.4 gives the detail about evaluation of traveler's willingness-to-pay travel related costs. Software implementation to simulate the proposed framework is described in section 3.5. Results and conclusion section are described in section 4 and 5. At the end of the paper, extensions are suggested.

\section{Literature Review}

Representation of the real systems via simulation has been applied to a broad range of topics in transportation sciences including simulation of vehicles or pedestrian flow, car following and lane changing models, route choice modeling, and traffic simulation. This is the key ingredient that drives most decisions in transportation planning and traffic operations. A few research studies that solve the transportation problems are discussed below;

The agent-based modeling (ABM) is used by Hussain et al. [1] to measure the individuals' behavior by applying direct interaction between agents in the carpooling process. Pel et al. [2] provide a simulation framework to model travelers' decisions regarding the selection of departure time, route selection and destination choice for evacuation. Luetzenberger et al. [3] introduce an approach that considers a driver's mind and examines the effect of environmental conditions. Authors planned to integrate the agent interactions necessary when carpooling. Rosswog et al. [4] proposed an algorithm to find user-equilibrium in simulation-based traffic network, which they further use to find shortest travel time path in street networks.

Another category of research efforts, mechanisms underlying the schedule construction and schedule adaptation processes are investigated. Knapen, et al. [5] enables explicit modeling of the information flow between traffic information services and travelers. It combines macroscopic traffic assignment with microscopic simulation of individuals and offers a framework to investigate algorithms for rescheduling at a large scale. The authors used marginal utility that monotonically decreases with activity duration, and a monotonically converging relaxation algorithm to efficiently determine the new activity timing. Recker [6] and Gan and Recker [7] present a mixed integer programming formulation of the Household Activity Rescheduling Problem, an extensively elaborated rescheduling model that has been applied to a small amount of individuals suffering from a pre-specified loss of time. The idea is that while planning, people solve a MILP (mixed integer linear program) and the examples given show that realistic schedules are produced. However, the large numbers of constraints required and the level of detail does not allow for large-scale deployment. Nandam [8] lists the main characteristics of Computational Process Models (CPM) and their 
assumed shortcomings. They describe a data collection method to acquire data to uncover the (re) planning process. The method used is similar to the one described in Weis et al. [9] who report the frequency of activity compression due to increased travel duration but not the amount of compression for several activity types. Van Bladel et al. [10] point out the difficulties to estimate the utility function parameters and shows the S-shaped dependency of the utility on the time gap since the preceding execution of a same activity

Another set of research efforts categorizes as calculation of value of time and willingness-to-pay the travel related cost. Ettema et al. [11] evaluated the traveler's responses to road pricing schemes by modeling the marginal utility for activities in two parts. Duration-based marginal utility is modeled using a logarithmic function while time-of-the-daydependent marginal utility is described by using Cauchy function. Jenelius et al. [12] analytically derive the optimal timing in a schedule composed of three activities and two trips. The authors analyze a model using marginal utility functions for each activity using piecewise sigmoid function where the magnitude of the function is bound by maximum and minimum value of time of the individual for specific activity. Joh et al. [13] provide schedule generation and dynamic activity travel rescheduling decisions. The Aurora model is based on S-shaped utility functions. The maximal utility value attainable for a given activity is given by the product of functions modeling the attenuation by start time, location, position in the daily schedule and time break since last execution of the activity. Nagel et al. [14] estimated the effect on traffic flows of willingness-to-pay the congestion cost by modeling the marginal cost and marginal utility ratios using MATSim; Multi-agent Transportation Simulation tool.

Kouwenhoven et al. [15] used stated preference surveys to calculate the value of time for passenger transport. Such methods provide value of time related with single trip without considering trip-based constraints. Similar methods of Value of time calculation have been reported by Baqueri et at. [16], Hess et al. [17], Fezzi et al. [18] and Meunier and Quinet [19] where authors used multinomial logistic regression to find travel time and cost coefficients using SP data which result in value of time.

\section{Proposed model to determining changes in activity duration}

Individuals plan their daily activities to achieve maximum utility of the time by keeping the travel cost minimum. The travel cost involved in activity planning is only perceived cost i.e. fuel cost, toll cost and/or congestion cost. Individuals do not take into account the past investment cost in their daily planning. In this work, congestion cost along with fuel cost is taken into account in an optimization of travel start time with constraints of maximization of time utilization and minimization of the total cost involved with trips. Daily schedules of the individuals are rescheduled in order to fulfill the cost and utility constraints. The proposed rescheduling is considered day-ahead planning only without feeding the planning information back to the transportation network and using the updated state of network back to planning procedure.

Congestion charges are applied with flat rate to all car drivers travelling during the charging period. Each individual, to whom congestion cost applied, intend to reschedule his or her trips that overlap with congestion charging period. Rescheduling of trips requires a tradeoff among difference in value of reallocated time and saved cost. Re-planning of time for one trip may have consequences in preceding and succeeding activities as well because utilization of time may differ from the original one and activity participation may also be changed.

To test above described hypothesis, sigmoid utility is modeled for each activity. Total utility of the schedule is compared for the original and rescheduled cases along with saved travel cost in order to calculate the individual's willingness-to-pay. In following sections, the theoretical model is described to calculate the utility of the schedule. This is followed by a description of the method to reschedule the original plan and the way to evaluate the utility to cost ratio. At the end of this section, the software implementation used to simulate the proposed evaluation framework is explained.

\subsection{Theoretical framework}

The framework modeled in this work postulates that individuals take part in daily activities and make trips to travel between activity locations. A given sequence of activities constitutes the daily schedule for each individual. Each activity has a preferred start time, duration and location. The initial daily schedules of the whole population of study area are generated using the activity-based model FEATHERS [20] and are considered to be optimal because of revealed preference. Feathers schedules are derived from household travel survey using data mining techniques. Therefore, revealed preference information is used. It is assumed that individuals try to maximize utility and hence the majority of the schedules can be assumed to be optimized in practice. Optimal schedule connotes that the individual 
considers activity start time and duration optimized which yields maximum satisfaction required by activity participation. The satisfaction is the maximum benefit earned by the individual by spending time at different activities and trips during the day and it is represented by schedule utility $U$. Total utility of the schedule $U$ is the sum of utility of all activities in the schedule. The utility gained by spending a unit of time on any activity is expressed using a bellshaped marginal utility, which is described in section 3.1.1. Such models have been formulated previously by Ettema and Timmermans [21], Zhang et al.[22] and Jenelius et al.[12]. However all previous models only formulate the fixed number of activities or using parameters that do not take the complete schedule into account. In this work, a procedure is described to derive the parameters involved in marginal utility function. Suppose that a schedule consists of $n$ activities where $n-1$ trips are required to travel between $n$ activities locations. Each activity $i$ starts at $t_{i}^{S}$ and ends at $t_{i}^{e}$ time of day. Each trip is $T_{i}$ duration long, which starts from the end time $t_{i-1}^{e}$ of the previous activity $i-1$ and ends at start time $t_{i}^{S}$ of the succeeding activity $i$. Utility $u_{i}(t)$ of each activity is calculated by integrating the marginal utility of the activity from its starting time $t_{i}^{s}$ to the end time $t_{i}^{e}$, hence, the utility is a function of duration $D_{i}$ of the activity. The total utility $U$ of the schedule is the sum of the utilities of all activities as shown in formula (3.2), where $v_{i}(t)$ is the marginal utility of the activity $i$.

$$
\begin{gathered}
\boldsymbol{D}_{\boldsymbol{i}}=\boldsymbol{t}_{\boldsymbol{i}}^{\boldsymbol{e}}-\boldsymbol{t}_{\boldsymbol{i}}^{\boldsymbol{S}} \\
u_{i}\left(D_{i}\right)=\int_{t=t_{i}^{e}}^{t_{t=t_{i}^{s}}^{s}} v_{i}(t) d(t) \\
U=\sum_{i=1}^{n} u_{i}\left(D_{i}\right)
\end{gathered}
$$

\subsubsection{Bell shaped marginal utility}

Marginal utility $v(x)$ is expressed as difference of two logistic functions, which are ' $\Delta$ ' units of duration apart from each other and have equal steepness. As shown in Fig. 1, solid line denotes the marginal utility $v(x)$ that is achieved by taking difference in two logistic functions $L_{1}(x)$ and $L_{2}(x)$ expressed by dotted lines. Difference in two logistic functions having equal steepness results in bell-shaped function, as expressed in equation (3.6), for which different examples are shown in Fig. 2. Hence, resulting bell function has three parts 1) warm-up part, 2) constant part (nearly constant for sufficient larger $\alpha$ values), and 3) cool-down part. Marginal utility function having warm-up and cooldown parts is advantageous over monotonic decreasing marginal utility described by Knapen et. al.[23] to model the effect of switching between tasks. In reality, marginal utility can be described in many ways. However, benefit yielded by activity participation for most activities saturates after a period which is warming-up phase. Similarly, at the end of activity, marginal utility gradually decreases and eventually reaches at the minimum point, which is cool-down phase. Examples of such marginal utility are presented in in Fig. 2, where four curves show the examples of marginal utility functions having different steepness and duration. Steepness of the marginal utility curve is expressed using $\alpha$ and the duration of the activity is expressed using the $\Delta$ variable. In the shown curves, center of the increasing slope occurs at the $\Delta=0$ and center of decreasing slop occurs the specific duration value of each curve. It is important to note here that marginal utility function described here does not depend on time of the day but only depends on time duration.

$$
\begin{gathered}
L(x)=\frac{1}{1+e^{-\alpha x}} \\
v(x)=k \cdot[L(x)-L(x-\Delta)] \\
v(x)=k \cdot\left[\frac{1}{1+e^{-\alpha x}}-\frac{1}{1+e^{-\alpha(x-\Delta)}}\right]
\end{gathered}
$$




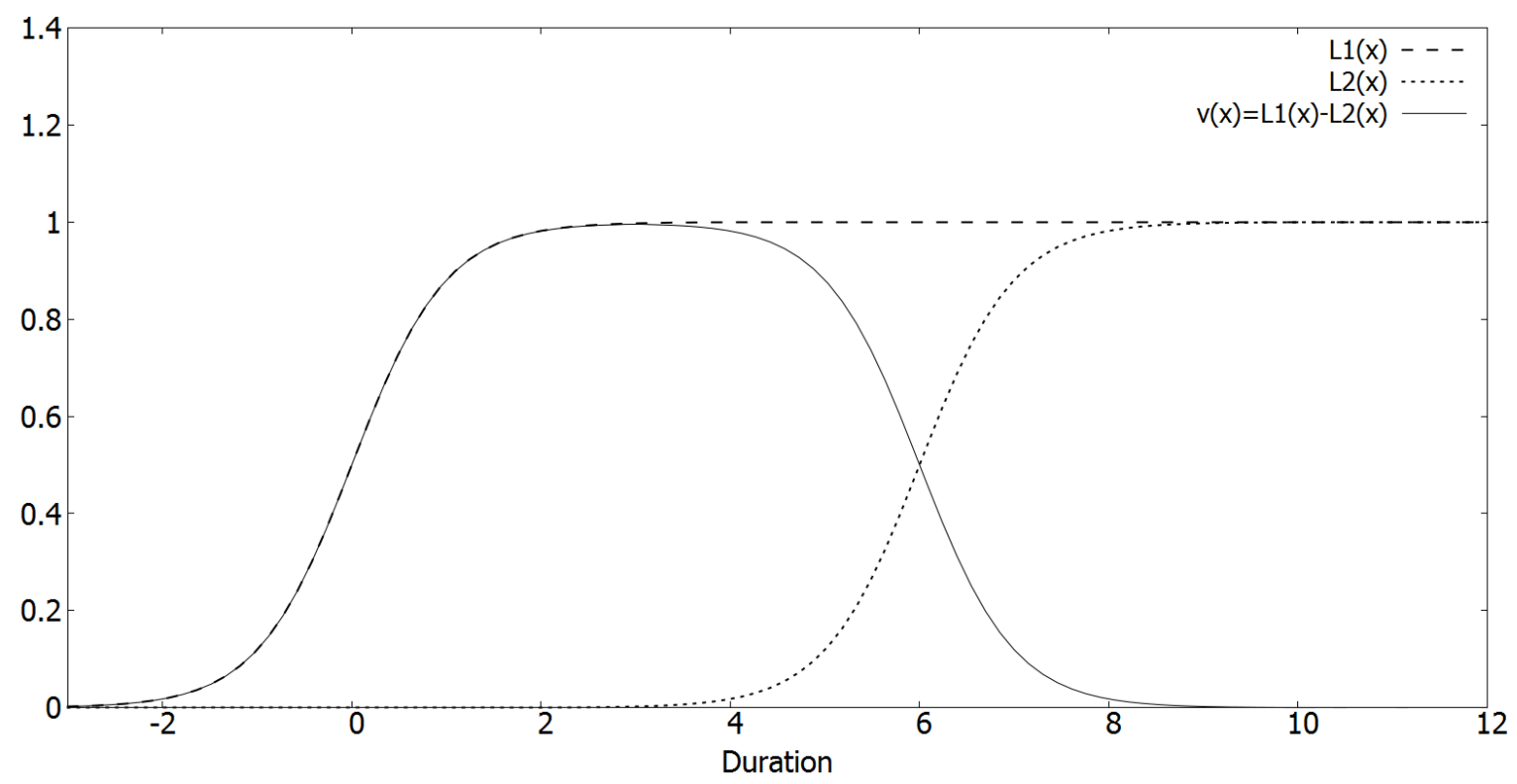

Fig. 1 Marginal utility function achieved by difference in two logistic functions

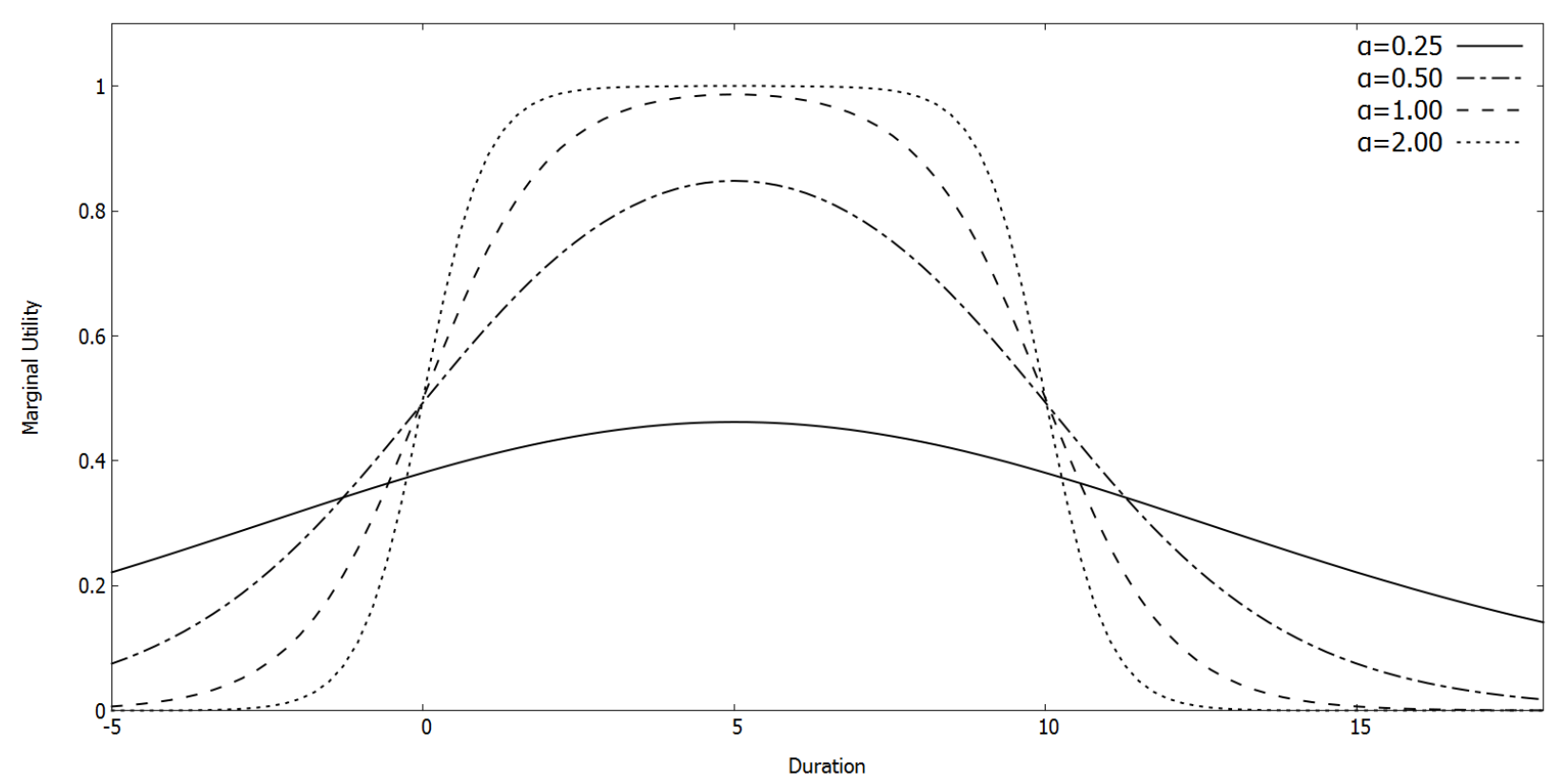

Fig. 2 Examples of bell-shaped marginal utility curves having different $\alpha$ with $\Delta=10$

\subsubsection{Sigmoid Utility}

Utility achieved by integrating the bell shaped marginal utility results in sigmoid function. Sigmoid utility $u_{i}$ of a given activity $i$ is a function of duration $\Delta$ and steepness factor $\alpha$. Let $\Delta_{i}$ be the typical/optimal duration described in section 3.1.5 and $D_{i}$ is the observed/actual duration of an activity $i$ where $\Delta_{i}=t_{i}^{t y p, e}-t_{i}^{t y p, s}$ and $D_{i}=t_{i}^{o b s, e}-t_{i}^{o b s, s}$. Then, utility is given by equation (3.8) with a function of duratition ${ }_{i} D_{i}$.

$$
u\left(t_{i}^{o b s, s}, t_{i}^{o b s, e}\right)=k \cdot \int_{t=t_{i}^{o b s, s}}\left[L(t)-L\left(t-\Delta_{i}\right)\right] d t
$$




$$
u\left(D_{i}\right)=\frac{k}{\alpha} \cdot\left[\ln \frac{1+e^{\alpha D_{i}}}{2}-\ln \frac{1+e^{\alpha\left(D_{i}-\Delta_{i}\right)}}{1+e^{-\alpha \cdot \Delta_{i}}}\right]
$$

Examples of utility are shown in Fig. 3 where four curves represent four different utility functions. All curves of utility shown in the example have $\Delta=10$ but four different values of $\alpha$. Sigmoid utility saturates early with higher value of $\alpha$.
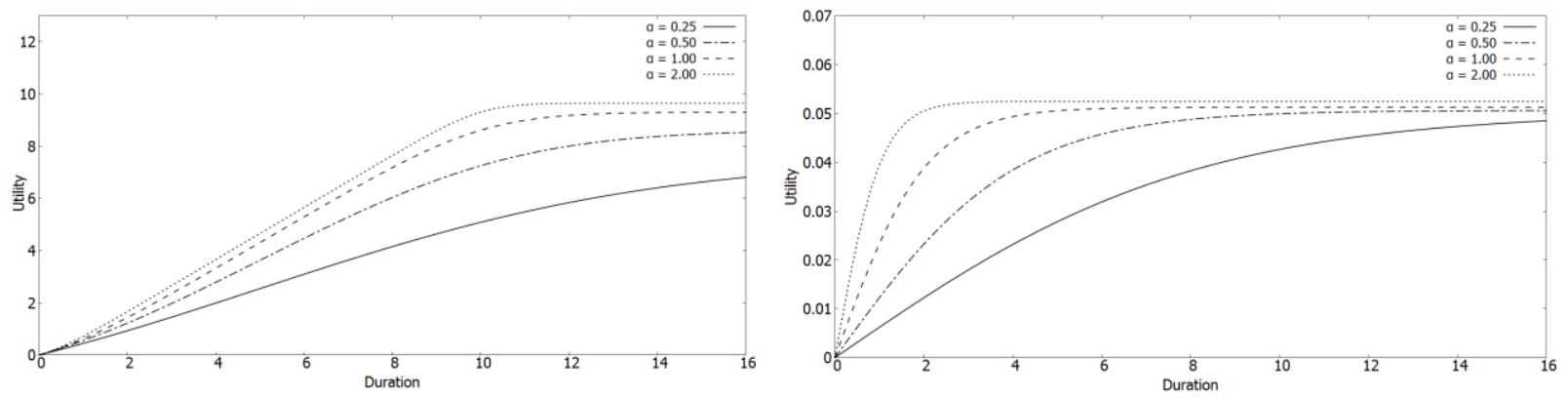

Fig. 3 Examples of utility curves with function of duration and different values of $\alpha$ and $\Delta$ : left Fig. with $\Delta=10$ and right Fig. with $\Delta=0.1$

\subsubsection{Determination of parameters}

To derive the parameters of marginal utility it is assumed that initial schedules created by activity-based model are optimal. Hence, marginal utility function is attuned to gain the utility $U<U_{\max }$ where $U_{\max }$ is maximum obtainable utility. Parameters of marginal utility are determined separately for each schedule without any dependency on other schedules. Hence, there is no dependency between individuals.

\subsubsection{Determination of Delta $(\Delta)$}

During schedule simulation, schedules are adapted, as described in section 3.3, where the duration of activities can change. New duration is termed as observed/actual duration $D_{i}$ of the activity during schedule simulation. Observed/actual can be longer or shorter than optimal/typical duration of the activity. If $t_{i}^{o b s, s}$ is the observed/actual start time and $t_{i}^{o b s, e}$ is the observed/actual end time of an activity $i$ then observed/actual duration for the marginal utility function of that activity is calculated as follow: $D_{i}=t_{i}^{o b s, e}-t_{i}^{o b s, s}$.

All activities in the schedule have typical duration of activity participation. Any change (positive or negative) in the duration of an activity will decrease the utility of complete schedule. We assume that the typical duration delta $(\Delta)$, which defines the width of the bell function, is the observed duration of the activity in the schedule. If $t_{i}^{t y p, s}$ is the optimal/typical start time and $t_{i}^{\text {typ,e }}$ is the optimal/typical end time of an activity $i$ then typical duration or delta $(\Delta)$ for the marginal utility function of that activity is calculated as follows: $\Delta_{i}=t_{i}^{t y p, e}-t_{i}^{t y p, s}$.

\subsubsection{Determination of Alpha $(\alpha)$}

As described earlier, parameters of marginal utility function are determined to gain a utility $U$, which is always lower than maximum utility $U_{\max }$ so that $U / U_{\max }$ will always be less than one.

For each activity in the simulated schedule, the activity duration is assumed to equal the typical duration $\Delta$ for the specific case (individual, activity type, day-of-week, time-of-day). Then $\Delta=t_{i}^{o b s, e}-t_{i}^{o b s, s}$. In this case, equation (3.8) leads to

$$
u(\Delta)=\frac{k}{\alpha} \cdot\left[\ln \frac{1+e^{\alpha \cdot \Delta}}{2}-\ln \frac{1+e^{\alpha \cdot 0}}{1+e^{-\alpha \cdot \Delta}}\right]
$$




$$
u(\Delta)=\frac{k}{\alpha} \cdot\left[\frac{2+e^{\alpha \cdot \Delta}+e^{-\alpha \cdot \Delta}}{4}\right]
$$

An asymptotic upper boundary for the utility is given by

$$
u_{\max }=\Delta \cdot v_{\max }
$$

The maximum marginal utility $v_{\max }$ occurs at $\frac{t^{s}+t^{e}}{2}$ which is equal to $t^{s}+\frac{\Delta}{2}$, hence following is derived from equation (3.5)

$$
\begin{gathered}
v_{\max }=v\left(\frac{\Delta}{2}\right) \\
v_{\max }=k \cdot\left[\frac{1}{1+e^{-\alpha \cdot \frac{\Delta}{2}}}-\frac{1}{1+e^{\alpha \cdot \frac{\Delta}{2}}}\right]
\end{gathered}
$$

Note that $v_{\max }$ decreases with decreasing $\alpha$. The utility $u_{\max }$ is realized for $\alpha \rightarrow \infty$. The relative utility that is defined as the ratio between actual and maximum utility can be expressed as:

$$
\begin{gathered}
r(\alpha)=\frac{u(\Delta)}{v_{\max } \cdot \Delta} \\
r(a)=\frac{1}{\alpha \cdot \Delta} \cdot \ln \left[\frac{2+e^{\alpha \cdot \Delta}+e^{-\alpha \cdot \Delta}}{4}\right]\left[\frac{1}{1+e^{-\alpha \cdot \frac{\Delta}{2}}}-\frac{1}{1+e^{\alpha \cdot \frac{\Delta}{2}}}\right]^{-1} \\
r(a)=\frac{1}{\alpha \cdot \Delta} \cdot \ln \frac{2+e^{\alpha \cdot \Delta}+e^{-\alpha \cdot \Delta}}{4} \cdot \frac{2+e^{\alpha \cdot \frac{\Delta}{2}}+e^{-\alpha \cdot \frac{\Delta}{2}}}{e^{\alpha \cdot \frac{\Delta}{2}}-e^{-\alpha \cdot \frac{\Delta}{2}}}
\end{gathered}
$$

1. The relative attainable maximum utility for $\Delta=10$ is shown in Fig. 4. In order to understand the presence of a local minimum, look at Fig. 2. Lower $\alpha$ values result in lower marginal utility (and hence in lower utility for a given duration). However, the marginal utility tends to approach a constant value for low $\alpha$ values more quickly so that for a given $\Delta$ the marginal utility $v$ approaches its maximal value $v_{\max }$ over a large part of the activity execution interval. As a result, the relative maximum attainable utility is high although the absolute utility attained might be low. 


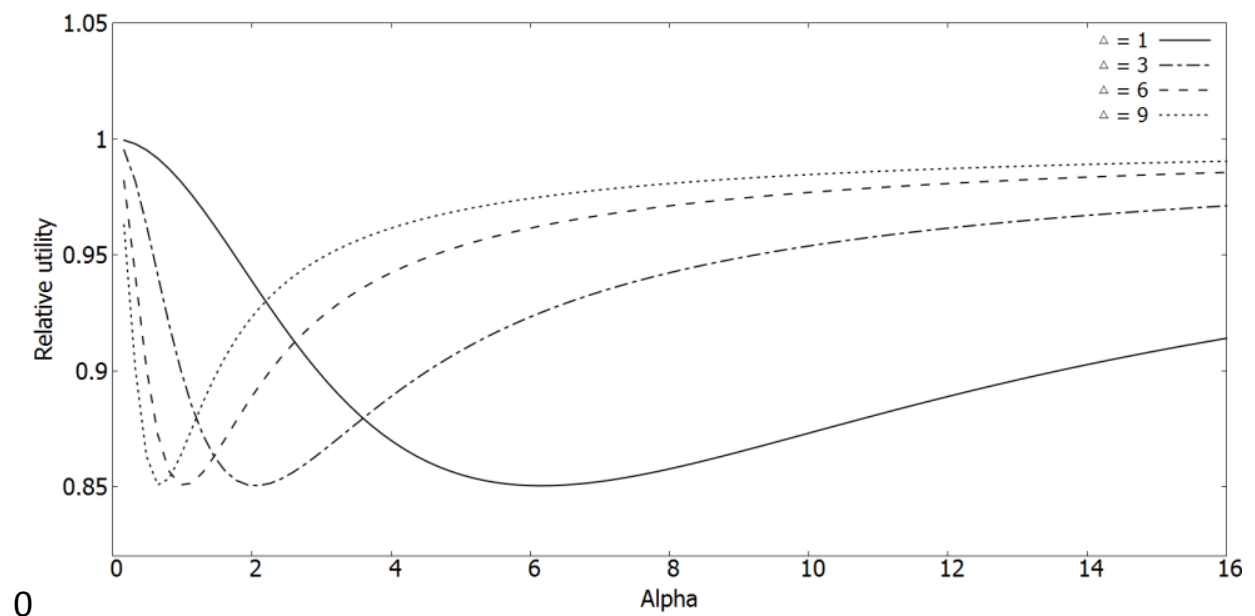

Fig. 4 Curves of relative utility as a function of awith different values of $\Delta$

2. In order to determine the value for alpha $(\alpha)$ it is assumed that the individual reaches a sufficiently large fraction $f$ of the maximum attainable utility (because the activity was observed and hence one can assume that it would not have been executed if the resulting utility were low. Therefore, out of the two available solutions of the equation (3.17), the larger alpha $(\alpha)$ value is to be retained. A Newton-Raphson iteration is used since equation (3.16) seems not to be tractable analytically.

$$
r(a)=f
$$

\subsection{6 $K$ values}

After some analytical exploration of the marginal utility function of two adjacent activities at the activity switching time, it is found that $\mathrm{k}$ values of all activities in the schedule will be equal. Starting with same argument that initial schedules are optimal and hence all consecutive activities in a schedule have equal marginal utility at time of switching from one activity to the next activity as shown by Knapen, et al.[5]. Assume two activities $a_{1}$ and $a_{2}$ are planned consecutively. Activity $a_{1}$ starts at $t_{0}$ and ends at $t_{1}$ while activity $a_{2}$ starts from $t_{1}$ and ends at $t_{2}$. The respective activity durations are $\Delta_{1}=t_{1}-t_{0}$ and $\Delta_{2}=t_{2}-t_{1}$. Hence, marginal utilities of both activities will be equal at $t_{1}$ at which activity switching occurs.

$$
v_{1}\left(t_{1}\right)=v_{2}\left(t_{1}\right)
$$

Equation (3.6) is inserted on both sides of equation (3.18), which leads to

$$
\begin{aligned}
& k_{1}\left[\frac{1}{1+e^{-\alpha_{1} \cdot \Delta_{1}}}-\frac{1}{2}\right]=k_{2}\left[\frac{1}{1+e^{-\alpha_{2} \cdot \Delta_{2}}}-\frac{1}{2}\right] \\
& \frac{k_{2}}{k_{1}}=\frac{1+e^{-\alpha_{2} \cdot \Delta_{2}}-e^{-\alpha_{1} \cdot \Delta_{1}-\alpha_{2} \cdot \Delta_{2}}-e^{-\alpha_{1} \cdot \Delta_{1}}}{1-e^{-\alpha_{2} \cdot \Delta_{2}}-e^{-\alpha_{1} \cdot \Delta_{1}-\alpha_{2} \cdot \Delta_{2}}+e^{-\alpha_{1} \cdot \Delta_{1}}}
\end{aligned}
$$

The assumption that the relative utility attained is identical for all activities in the schedule leads to the conclusion that the product $\alpha \Delta$ is the same for all activities. This follows from equation (3.16) when $r(\alpha)$ is given. Hence

$$
\alpha_{1} \Delta_{1}=\alpha_{2} \Delta_{2}=\alpha_{3} \Delta_{3}=\cdots=\alpha_{n} \Delta_{n}
$$


Substitution of equation (3.21) into equation (3.20) yields

$$
\frac{k_{2}}{k_{1}}=1
$$

Hence, for all activities in the schedule

$$
\frac{k_{1}}{k_{2}}=\frac{k_{2}}{k_{3}}=\cdots=\frac{k_{n-1}}{k_{n}}
$$

\subsection{Travel cost}

Travel costs $\left(t_{\text {cost }}\right)$ incurred to the traveler are fuel cost $\left(F_{\text {cost }}\right)$ and congestion charging $\left(c_{\text {cost }}\right)$. Details about congestion charging are described in section 3.2.1. Fuel cost is calculated as fuel consumption rate $F_{\text {rate }}^{\text {cons }}$ multiplied by the distance travelled by car $\left(t_{d i s}^{c a r}\right)$.

$$
\begin{aligned}
t_{\text {cost }} & =c_{\text {cost }}+F_{\text {rate }}^{\text {cons }} \cdot t_{\text {dis }}^{\text {car }} \\
F_{\text {rate }}^{\text {cons }} & =30[\text { Eurocent } / \mathrm{km}]
\end{aligned}
$$

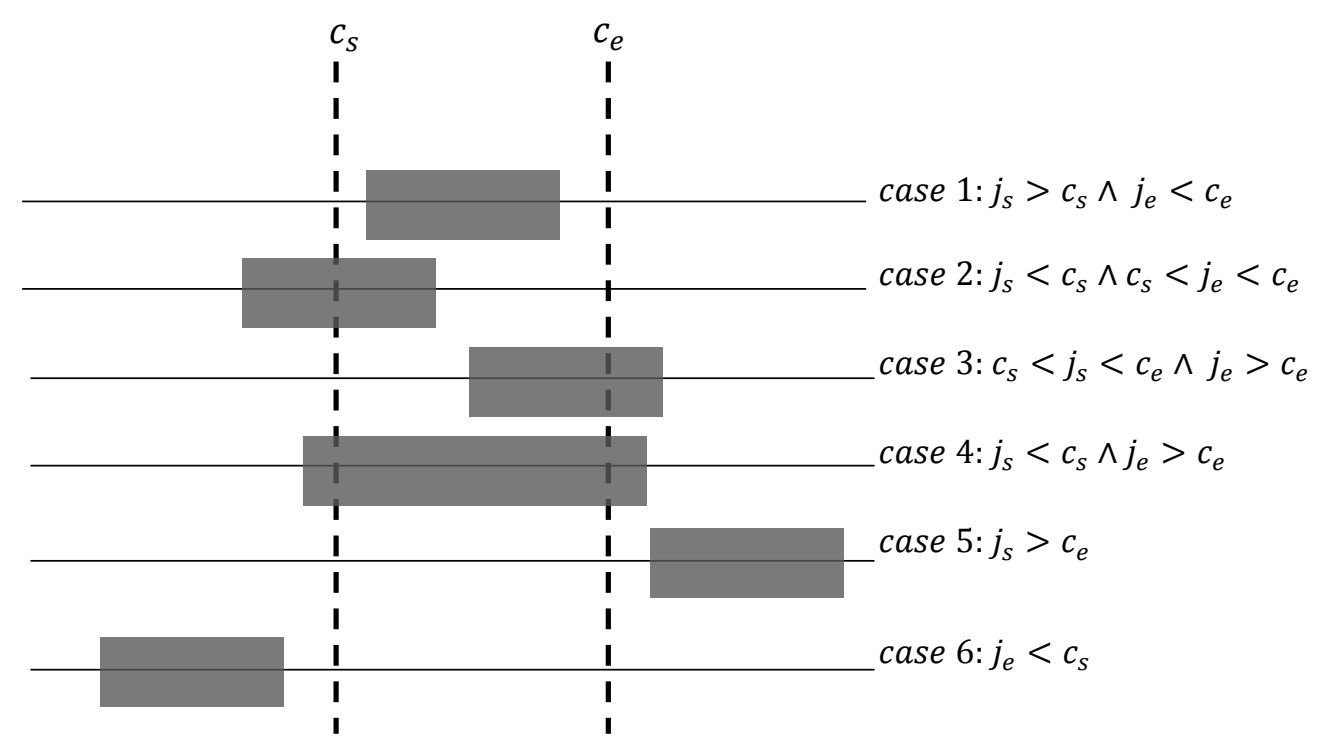

Fig. 5 Possible ways of overlap between congestion period and a trip

\subsubsection{Congestion period and congestion rates:}

A congestion profile is introduced for the morning peak for every car traveler. The congestion period starts from 07:00 am and ends at 09:00 am. Every car traveler, having active travel during the congestion period is charged for congestion. Congestion charging $c_{\text {rate }}$ charged as Eurocents per kilometer. Hence, congestion cost $c_{\text {cost }}$ is calculated depending on the number of kilometers travelled during the congestion period.

Overlap between travel and congestion periods can occur in four ways as shown in Fig. 5. Let $j_{s}$ and $j_{e}$ denote journey start and end time while $c_{s}$ and $c_{e}$ are start and end time of congestion period. Travel and congestion period can occur in several ways. As shown in the figure, overlap between travel and congestion period is possible only as described in 
cases 1 to 4 . If congestion and travel periods occur as shown in cases 5 and 6 , the overlap period between them will be zero.

Let $T^{p}$ denotes the intersection of the travel and congestion periods, $T_{s}^{p}$ denote the start time and $T_{e}^{p}$ the end time of the period of overlap. Calculation of starting and end times of the overlap period can be generalized as follows:

$$
\begin{gathered}
T_{s}^{p}=\operatorname{Max}\left(j_{s}, c_{s}\right) \\
T_{e}^{p}=\operatorname{Min}\left(j_{e}, c_{e}\right) \\
T_{d u r}^{P}=\operatorname{Max}\left(0, T_{e}^{P}-T_{s}^{P}\right)
\end{gathered}
$$

where $T_{d u r}^{p}$ is the duration of the overlap period. There will be an overlap between travel and congestion period if $T_{d u r}^{p}>0$ (cases 1 to 4$)$, and no overlap when $T_{d u r}^{p}=0$ (cases 5 and 6 ).

If a non-zero overlap between travel and congestion period is found, the distance travelled during the congestion period is required to calculate the congestion cost applied to the traveler. The only information available about travel is starting time, travel duration, travel distance, starting location and ending location. Exact route followed from starting location to the destination is also unknown. In this scenario, to calculate the exact distance travelled during overlap period is impossible. The distance is estimated as follow:

Assume that the individuals travel at uniform speed throughout the trip. If $\operatorname{dis}(t)$ is the trip distance, $d u r(t)$ is the trip duration, then distance travelled $T_{d i s}^{p}$ during congestion overlapping period $T_{d u r}^{p}$ can be found as follows:

$$
T_{d i s}^{p}=\frac{\operatorname{dis}(t)}{\operatorname{dur}(t)} \cdot T_{d u r}^{p}
$$

Hence, congestion cost is calculated as follows:

$$
\begin{gathered}
c_{\text {cost }}=T_{\text {dis }}^{p} \cdot c_{\text {rate }} \\
c_{\text {rate }}=10[\text { Eurocent } / \mathrm{min}]
\end{gathered}
$$

\subsection{Schedule adaptation}

Original daily schedules predicted by FEATHERS are assumed to be optimal (and hence results in maximum attainable utility); it incurs travel cost in terms of fuel cost but no congestion cost to the travelers. Congestion cost only applies to any traveler if he/she has any car trip during congestion period. Now we assume that any car trips which overlaps with congestion period is tried to shift up to $s_{\max }$ time units. The trip is shifted over time in order to bring it out of congestion period. This shift can result in lower travel cost as congestion cost will be lower than before (can also be zero if trip is completely shifted out of congestion period). Shift in any trip requires retiming of all activities and trips in the schedule; this is described in detail in section 3.3.2. Since activities are retimed, a new equilibrium state will emerge in the schedule. This adapted schedule may have less travel cost as compared to initial cost, but utility of the adapted schedule is always lower than the initial utility.

\subsubsection{Shift in trip}

Any car trip, which overlaps with the congestion period, is shifted either backwards or forwards in order to bring it out of congestion period. Decision of shifting the trip to the backward or forward depends upon to which direction required shift is smaller. The trip is shifted backwards if $s_{b w d}<s_{f w d}$, otherwise it is shifted forwards. Either way to shift, shifting value cannot be greater than the maximum possible shift $s_{\max }$. 
As shown in the Fig. 6, congestion period starts at $c_{s}$ and ends at $c_{e}$. There is a trip which overlaps with congestion period, it starts at $j_{s}$ and ends at $j_{e}$. Trip can be shifted out of the congestion period either shifting backwards for $s_{b w d}$ time units or to the forward for $s_{f w d}$ time units. In the example shown in this figure, shifting backwards requires less time shift then shifting forwards. Hence, backward shift is selected, but required shift is longer than maximum allowed shift. Hence, only a part of trip manages to avoid congestion period. Even in case of small part of trip shifts out of congestion period, it creates marginal difference of cost.

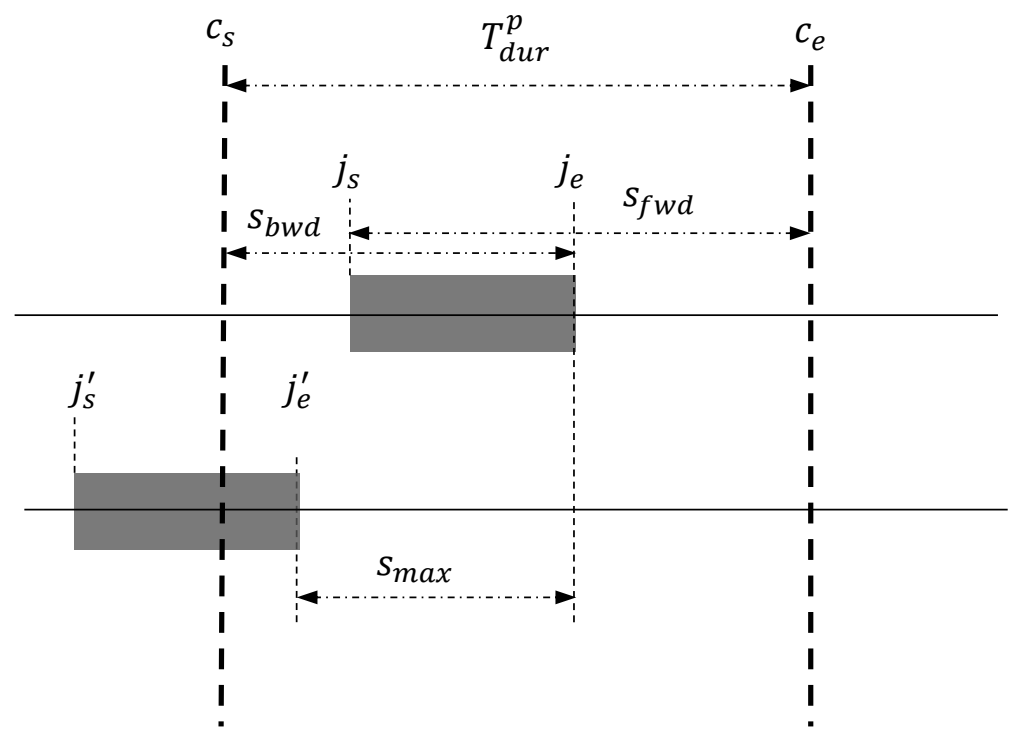

Fig. 6 Shift in a trip to avoid congestion charging

Shifting the trip adjusts the activity starting time and causes a change in duration of the activities between which the trip is enclosed in time. The activity that is compressed is under run, while the activity that is decompressed may get very low marginal utility due to overrun. This requires timing relaxation in the schedule while keeping the shifted trip fixed in place. This introduces an intermediate deadline in the schedule. The intermediate deadline is kept at a fixed point in time and the relaxation algorithm operates on the schedule in two independent parts. Time pressure and relaxation produced by compressing/decompressing activities are distributed among all activities in the respective parts of the schedule. An example is shown in the Fig. 7, where a schedule is presented (in row $\rightarrow 1$ ) having five activities (shown by white boxes) and four trips (shown by grey colored boxes) in between the activities. The second (from left) trip overlaps with the congestion period, and shifts backwards in time to avoid congestion cost as represented in row $\rightarrow 2$. This causes a compression in the second and a decompression in the third activity. The shifted trip shown with a bold line box on row $\rightarrow 3$, is fixed in time and its boundaries are treated as intermediate deadlines. All activities on both sides of the fixed trip are retimed to distribute the lost and gained time respectively. 


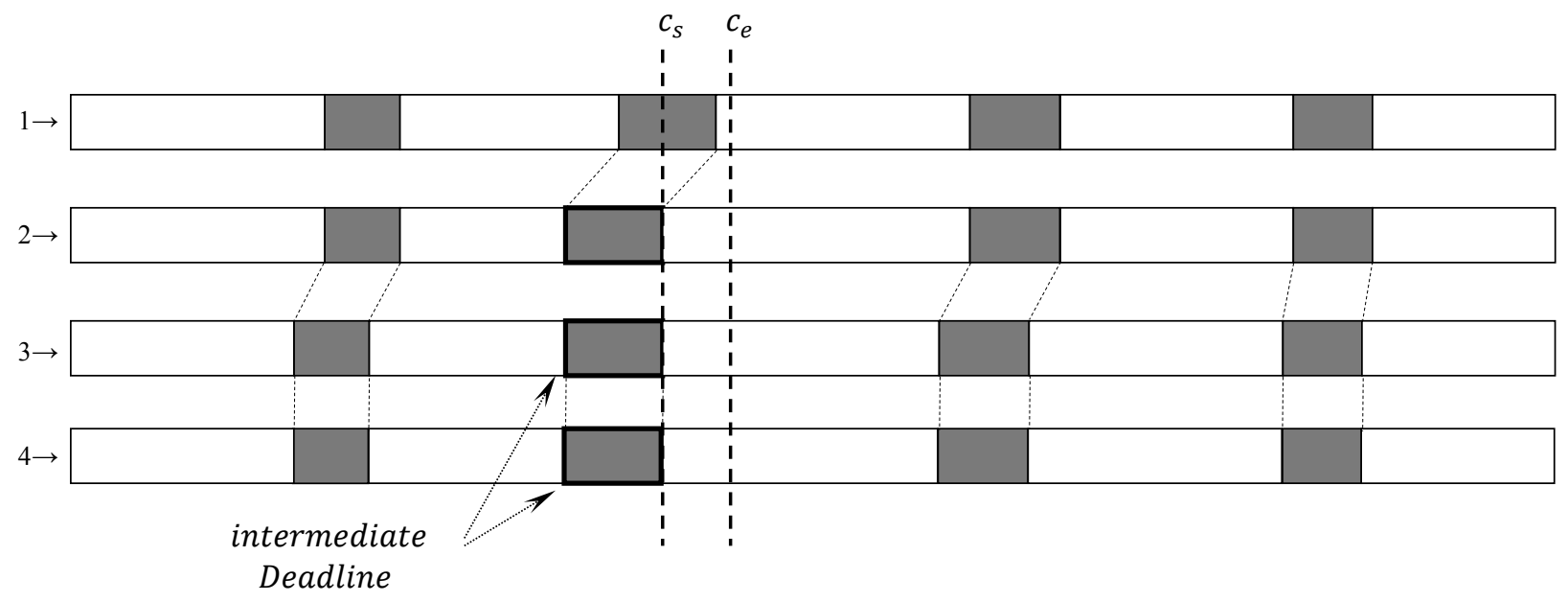

Fig. 7 Schedule (de) compression with intermediate deadline

During relaxation of activities in the schedule, start time and duration of each activity is recalculated. This is done by means of an iterative method, where two adjacent activities are evaluated in a pair. In each pair of two activities, swapping time from on activity to the other is recalculated by fixing their outer ends (start time of first activity and end time of second activity), and their duration values are updated according to the new time of swapping. Swapping between two adjacent activities should occur when the marginal utility value of first activity in cool-down part becomes equal to the marginal utility value of second activity at the warm-up part. This conforms to the hypothesis of equal marginal utility rule at activity swapping time. Using this rule, calculation of new activity starting time is described in section 3.3.2.

\subsubsection{Activity start time adjustment}

Consider two adjacent activities $a_{1}$ and $a_{2}$ have $d_{1}$ and $d_{2}$ units duration. Activity $a_{1}$ starts at $t_{0}$ and ends at $t_{1}$ where second activity $a_{2}$ starts at $t_{1}$ and ends at $t_{2}$. Travel from first activity to the second activity should occur at the point where the values of their marginal utilities are equal. The following method is used to calculate their new swapping time $t_{1}$ while keeping the outer ends $\left(t_{0}\right.$ and $\left.t_{2}\right)$ fixed.

$$
\begin{aligned}
v_{1}\left(d_{1}\right) & =v_{2}\left(d_{2}\right) \\
k_{1} \cdot\left[\frac{1}{2}-\frac{1}{1+e^{-\alpha_{1} d_{1}}}\right] & =k_{2} \cdot\left[\frac{1}{2}-\frac{1}{1+e^{-\alpha_{2} d_{2}}}\right]
\end{aligned}
$$

Since $k_{1}=k_{2}$, rewriting equation (3.34) yields,

$$
\begin{gathered}
\left(e^{-\alpha_{1} d_{1}}-1\right)\left(1+e^{-\alpha_{2} d_{2}}\right)-\left(e^{-\alpha_{2} d_{2}}-1\right)\left(1+e^{-\alpha_{1} d_{1}}\right)=0 \\
2 e^{-\alpha_{1} d_{1}}=2 e^{-\alpha_{2} d_{2}} \Rightarrow \alpha_{1} d_{1}=\alpha_{2} d_{2}
\end{gathered}
$$

Since $d_{1}=t_{1}-t_{0}$ and $d_{2}=t_{2}-t_{1}$

$$
\begin{gathered}
\alpha_{1}\left(t_{1}-t_{0}\right)=\alpha_{2}\left(t_{2}-t_{1}\right) \\
\Rightarrow t_{1}=\frac{\alpha_{1} t_{0}+\alpha_{2} t_{2}}{\alpha_{1}+\alpha_{2}}
\end{gathered}
$$




\subsection{Evaluation of change in cost to utility ratio}

Execution of a schedule has a travel cost and it produces some utility. Initial schedules are considered optimal; hence, they produce maximum utility. Introduction of congestion cost for a specific period of the day can increase the travel cost for an individual if there is any car trip during the congestion period. The method to evaluate the individual's decision to either accept the congestion cost and keeping the utility maximum or shift the trip to avoid the congestion cost leading to a utility drop is described in this section.

The proposed method to evaluate any individual's willingness-to-pay is based on hypothesis of minimizing the cost to utility ratio. Two scenarios are used to compare the ratio between cost and utility; (i) the optimal case (original schedule), and (ii) congestion avoidance described in section 3.3. The optimal case always results in maximum utility with some travel cost; it is used as the reference case. In the adapted case, utility of the schedule always decreases while cost either can decrease or increase. Travel cost of the schedule decreases if shifting a trip and following schedule relaxation does not bring any succeeding or preceding trip into the congestion period; otherwise, travel cost may increase.

So, ratio of cost to utility will decrease only if decrease in travel cost is higher than decrease in utility for the shifted period. On the other hand, the ratio of cost to utility will increase if the travel cost of the adapted schedule exceeds the reference cost or the decrease in the travel cost is lower than the decrease in utility for the shifted period. Therefore, an individual accepts to pay the congestion cost only if his/her ratio of cost to utility exceeds the corresponding value for the reference case. If the ratio of cost to utility drops, the individual will accept the adaptation of the schedule to save the congestion cost.

Table 1 Comparison of cost to utility ratios

\begin{tabular}{|c|l|}
\hline Indicator & Decision \\
\hline$\frac{C_{\text {shifted }}}{U_{\text {shifted }}} \leq \frac{C_{\text {init }}}{U_{\text {init }}}$ & Accepts adaptation in schedule to avoid congestion charging \\
\hline$\frac{C_{\text {shifted }}}{U_{\text {shifted }}}>\frac{C_{\text {init }}}{U_{\text {init }}}$ & Accepts to pay the congestion charging \\
\hline
\end{tabular}

Assume an individual $I$ has a schedule $S$ that consists of $n$ activities where at least one car trip has an overlap with congestion period. Initially schedule $S$ has the utility $U_{\text {init }}$ and a travel cost $C_{\text {init }}$, which is the fuel cost. In order to evaluate the individual I's willingness-to-pay the congestion cost, the trip which overlaps with congestion is shifted out of the congestion period and the activity timings in schedule $S$ are recomputed. Now cost $C_{\text {shifted }}$ and utility $U_{\text {shifted }}$ are calculated for the adapted schedule where $U_{\text {shifted }}$ is always less than $U_{\text {init }}$ while $C_{\text {shifted }}$ can be less than, equal to or greater than $C_{\text {init }}$. Their ratio is compared to evaluate the willingness-to-pay, which is summarized in Table 1.

\subsection{Implementation and Simulation}

A software simulation is implemented using Java to evaluate the theoretical model described above. Daily activity schedules of individuals, which are used as source input to feed the model are created by the FEATHERS activitybased model. The source information is used in the form of a list containing the complete information about each individual. In the simulation, an individual is represented by the person entity. Each person owns one schedule where a schedule is a list of episodes. An episode consists of a (possibly empty) trip and an activity. One FEATHERS prediction produces a schedule for each member of the considered population for one specific day only. A schedule starts from 3:00 AM in the morning and ends at 3:00 AM next day morning. The number of activities in the schedule for each person can vary and it is person specific. Each activity has a specific purpose, starting time, duration and location of activity execution. A trip is required to travel between two activity locations. Each trip has a specific mode of transport, travel duration and travel distance. As shown in Fig. 8 that represents the class diagram, the basic information about these entities is populated from FEATHERS is passed to the moduleManager. ParamCalibrator determines the parameters of the marginal utility function for each activity. 
CongestionProf entity contains the information about the congestion period and congestion rate. ScheduleAdapter module updates the timing of the trip that overlaps with the congestion period by (de)compressing the two activities one after and one before the trip. This module also contains the schedule relaxation algorithm that updates the timing of all activities in the schedule in two parts by fixing the time of the shifted trip. The framework contains the CUREvaluator, which calculates the total travel cost and total utility for initial schedule and adapted schedule. CUREvaluator compares the cost to utility ratio of the initial and adapted schedules to evaluate the willingness-to-pay the congestion cost.

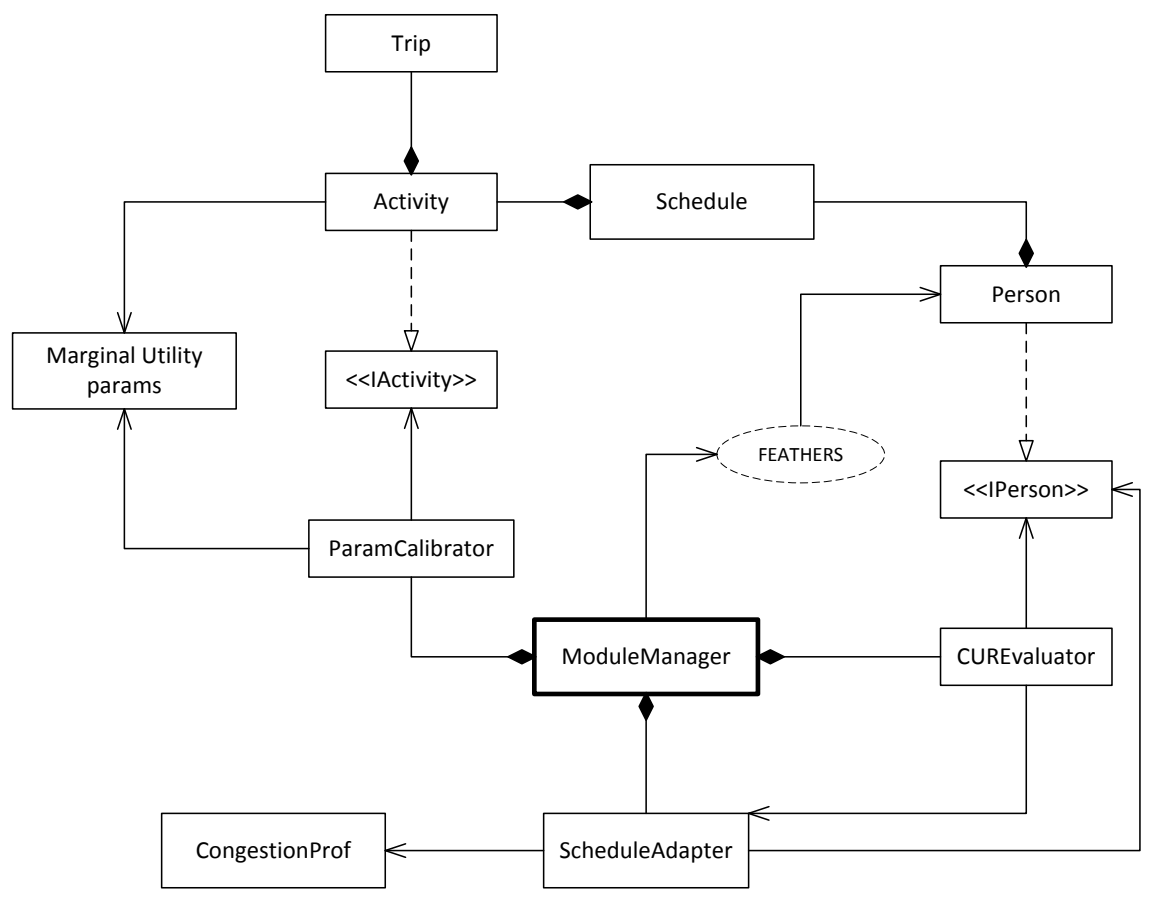

Fig. 8 Class diagram of the framework

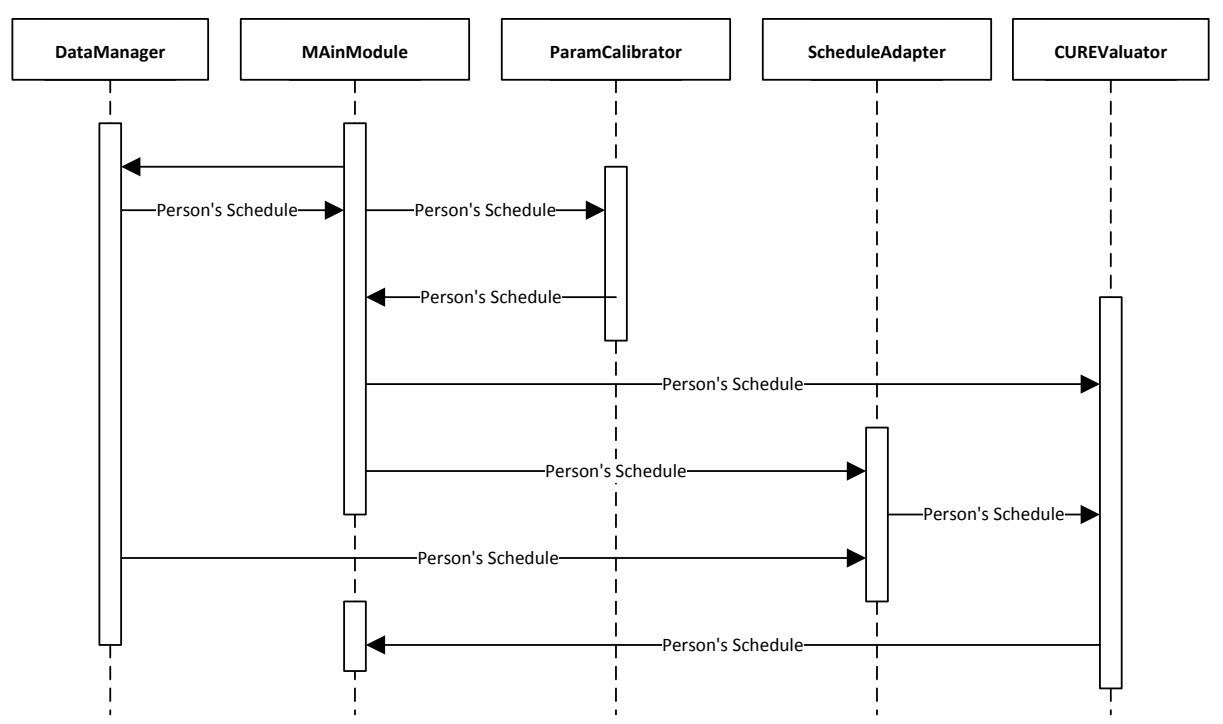

Fig. 9 Sequence diagram of flow of messages between framework entities 
Fig. 9 shows the sequence diagram to explain the interactions among the components of the framework. ModuleManager invokes the DataManager module to fetch the schedule of next person by reading the predictions made by FEATHERS. Then moduleManager calls the paramCalibrator module by passing this basic information to compute the marginal utility parameters using the basic information for each activity. ScheduleAdaptor fetches the congestion information from DataManager and using the information about personal schedule and congestion period it retimes all activities in the schedule by shifting trips out of the congestion period. ModuleManager sends the message to CUREvaluator to compare the utility and cost of original and adapted schedule.

\section{Experiment and Results}

The study area covers Flanders (Belgium). It is modeled by 2386 traffic analysis zones (TAZ) with an average area of about $5[\mathrm{~km}]^{2}$. TAZ are bundled into 319 municipalities. The population consists of 5.8 million individuals. FEATHERS predicts daily schedules of 2395514 individuals, out of which 805964 schedules contain at least one car trip during the considered day.

To compute the parameters of marginal utility, it is assumed that a fraction of maximum utility can be reached as described in equation (3.17). Fraction of utility to the maximum utility is used equal to $95 \%$. Hence alpha $(\alpha)$ values used in marginal utility function of each activity are calculated with $f=0.95$. During schedule adaptation, trips are shifted to avoid the congestion period by moving them either to the forward or to the backward, but trips are not allowed to shift more than a maximum value $S_{\max }$. This is based on carpooling research survey, which find that due to appointments, people do not shift their activities more than $30 \mathrm{~min}$ [24]. The evaluation presented here assumes that the maximum allowed shift is 30 minutes.

Fig. 10 shows the frequency distribution of difference in cost to utility ratio between adapted and initial schedules in a histogram. Out of $\mathbf{8 0 5 9 6 4}$ individuals who used car at least for one trip, 3.63\% individuals had an overlapping trip with a congestion charging period and found a different travel cost after schedule adaptation. Out of all individuals who found a different travel cost, $\mathbf{7 6 . 4 \%}$ individuals would adapt their schedule to avoid congestion cost and 23.6\% individuals were found willing to pay congestion cost rather than adapting their schedules.

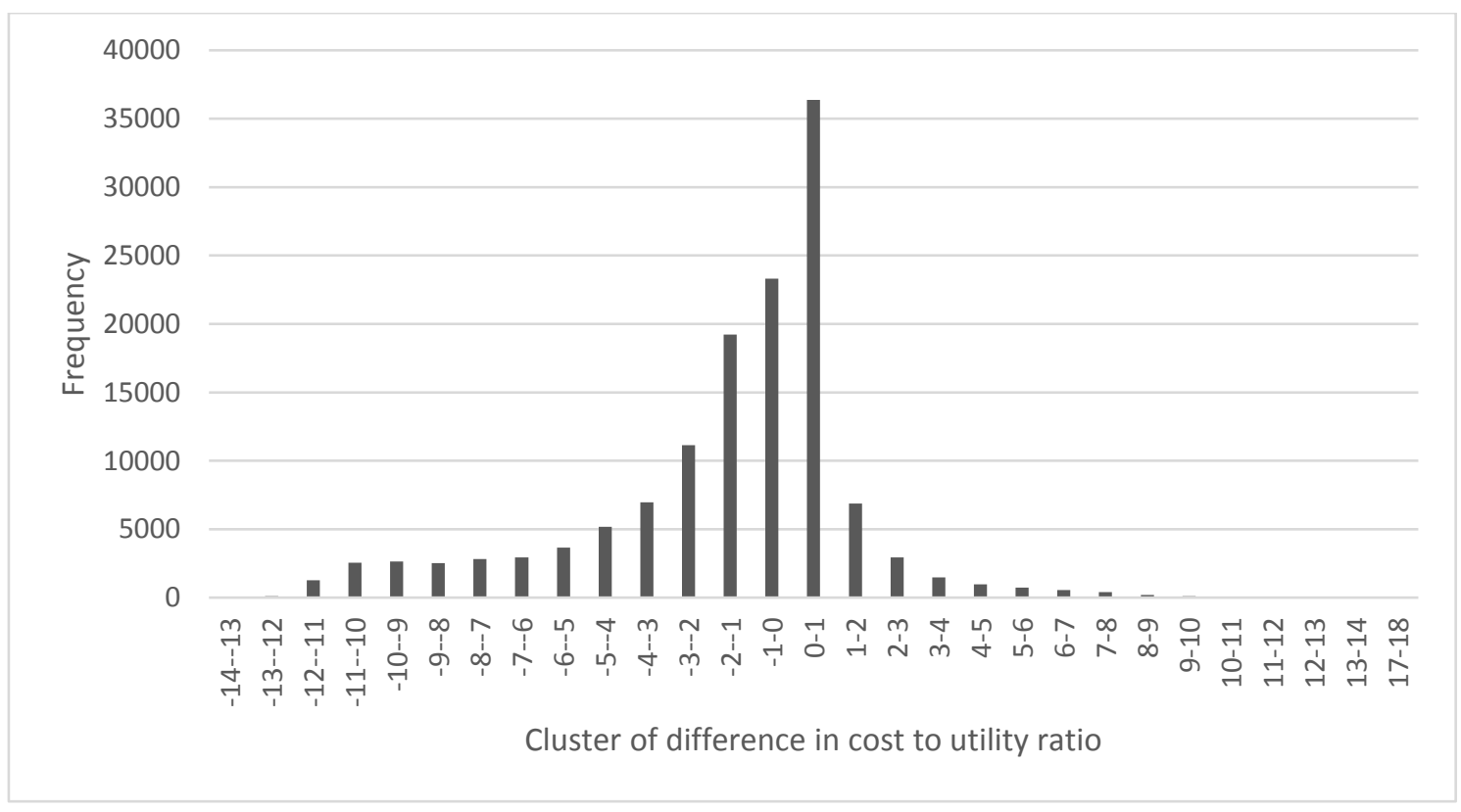

Fig. 10 Distribution of absolute difference in cost to utility ratio

Individual's behavior to decide the willingness-to-pay the congestion cost is predicted using the marginal utility function. The variables used in marginal utility function are determined by assuming that a certain fraction of maximum utility is attainable. The used fraction of maximum attainable utility determines the parameters used in marginal utility function and can have influence on behavior. To determine the influence of used fraction, a sensitivity test is carried 
out. To test the model's sensitivity, experiment is repeated using different values of relative utility $(f)$ that is described in equation (3.17). A range from $\mathbf{0 . 8 8}$ to $\mathbf{0 . 9 6}$ with step of $\mathbf{0 . 0 2}$ is used for relative utility to test the sensitivity of the model. Two variables used to sense the influence of relative utility are; 1) total congestion cost paid by the travelers, and 2) the fraction of the population that decide to pay the congestion cost. Fig. 11 shows the values of two variables for different values of relative utility. For different values of relative utility, the observable variation in both variables is very small.

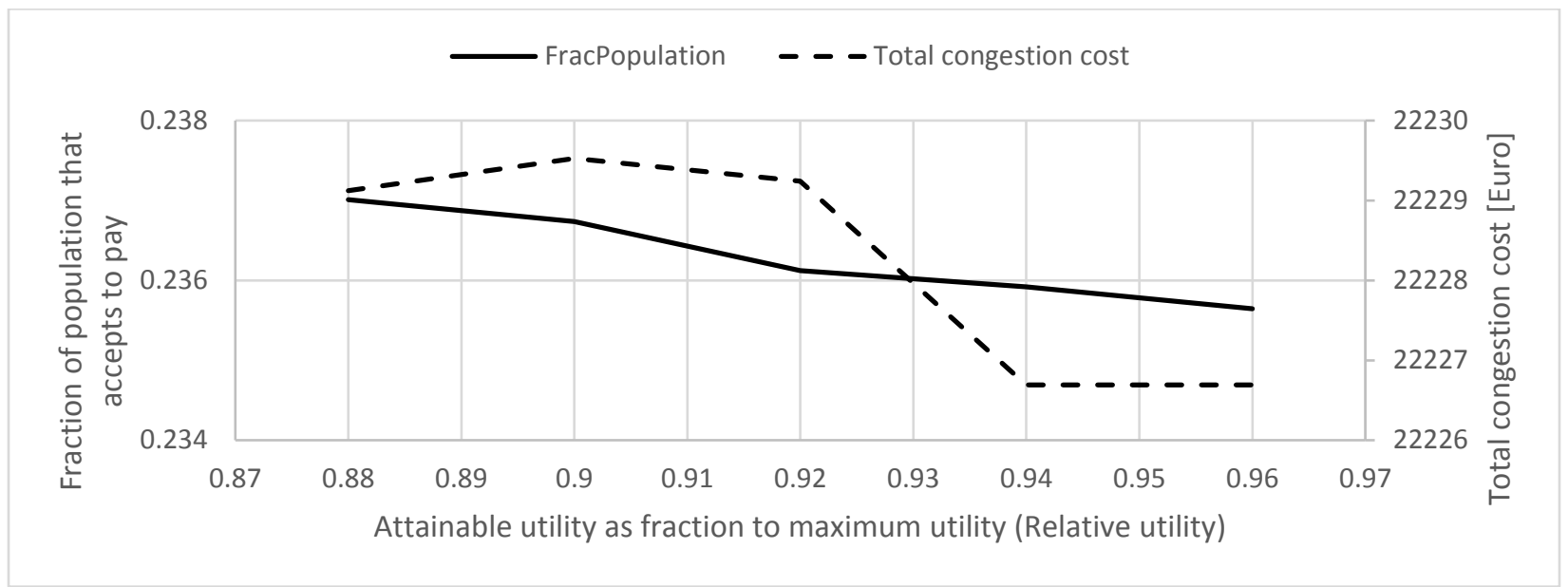

Fig. 11 Total congestion cost (dotted line) paid by the travelers and fraction of population (solid line) that decides to pay the congestion charges rather than rescheduling

\section{Conclusion}

In this work a model for rescheduling is presented, which can be useful to evaluate the effect of traveler's willingnessto-pay the congestion charges. Willingness-to-pay is evaluated by comparing the ratio of schedule utility and travel cost of original and adapted schedules. Individuals whose trips overlap the congestion charging period shift their trips in time if that decreases the cost per unit of utility. A new bell-shaped function is used to represent the marginal utility of the activities in the schedule, which results in sigmoid utility. The sum of sigmoid utilities of all activities in the schedule results in total schedule utility. A method is proposed to determine the parameters of marginal utility bellshaped functions using basic information about activity plans. In this study schedules predicted by an activity-based model FEATHERS were used as input. The simulated results show the percentage of travelers who would accept or reject to pay the travel cost.

\section{Future work}

Application of the proposed model with static input from congestion profile grants the travelers a freedom with given flexibility to adapt their schedule in order to save the congestion cost. At the individual level, adapted daily plans outperform the original ones but at the aggregated level a shifted traffic peak larger than the original one occurs. This is because the current model has no feedback loop using traffic assignment (i.e. the effect on travel time is not fed back). A feedback loop is required in an iterative procedure where individuals update their trips in the schedule with new travel duration. This can be achieved by adding a new module of traffic assignment that updates the state of the traffic network with adjusted travel times between locations. Hence, using updated schedules, individuals should recalculate their cost to utility ratio to evaluate their willingness-to-pay the congestion cost.

Schedule adaptation using monotonic decreasing marginal utility has been described by Knapen et al. [23] and a framework of large-scaled microsimulation using macroscopic information of traffic state is presented. Monotonically decreasing marginal utility connotes that during rescheduling, even in case of high time loss, individuals will prefer to execute the activity because marginal utility starts with highest value and decays monotonically. This may not be the case when bell-shaped marginal utility is used, since the marginal utility has a warm-up period, which cause different behavior due to heavy time loss. In case of high time pressure, individuals may prefer to drop an activity, which due to compression cannot reach its saturation period, to compensate the time loss of any other activity that is already in saturation period in order to maximize the total utility. 


\section{References}

[1] I. Hussain et al., "Organizational-based model and agent-based simulation for long-term carpooling," Future Gener. Comput. Syst., vol. 64, pp. 125-139, Nov. 2016.

[2] A. J. Pel, M. C. J. Bliemer, and S. P. Hoogendoorn, "A review on travel behaviour modelling in dynamic traffic simulation models for evacuations," Transportation, vol. 39, no. 1, pp. 97-123, 2012.

[3] M. Lützenberger, N. Masuch, B. Hirsch, S. Ahrndt, A. Heßler, and S. Albayrak, "Strategic Behavior in Dynamic Cities," in Proceedings of the 2011 Summer Computer Simulation Conference, Vista, CA, 2011, pp. 194-201.

[4] S. Rosswog, C. Gawron, S. Hasselberg, R. Böning, and P. Wagner, "Computational aspects in traffic simulation problems," Best Websim99 II Traffic Simul., vol. 17, no. 5, pp. 659-665, Mar. 2001.

[5] L. Knapen, M. Usman, A.-U.-H. Yasar, T. Bellemans, D. Janssens, and G. Wets, "Framework to Evaluate Rescheduling Due to Unexpected Events in an Activity-Based Model," presented at the Transportation Research Board 92nd Annual Meeting, Washington, USA, 2013, p. 21p.

[6] W. . Recker, "The household activity pattern problem: General formulation and solution," Transp. Res. Part B Methodol., vol. 29, no. 1, pp. 61-77, Feb. 1995.

[7] L. P. Gan and W. Recker, "A mathematical programming formulation of the household activity rescheduling problem," Transp. Res. Part B Methodol., vol. 42, no. 6, pp. 571-606, Jul. 2008.

[8] S. Nandam, A Data Collection Framework for Exploring the Dynamic Adaptation of Activity-travel Decisions. University of Wisconsin-Madison, 2012.

[9] Claude Weis, Christoph Dobler, and Kay W. Axhausen, "A Stated Adaptation Approach to Surveying Activity Scheduling Decisions," in Transport Survey Methods: Best Practice for Decision Making, 0 vols., Emerald Group Publishing Limited, 2013, pp. 569-590.

[10] K. Van Bladel, T. Bellemans, G. Wets, T. Arentze, and H. Timmermans, "Fitting S-shaped activity utility functions based on stated-preference data," presented at the : 11th International Conference on Travel Behaviour Research, Kyoto, 2006.

[11] D. Ettema, O. Ashiru, and J. Polak, "Modeling timing and duration of activities and trips in response to roadpricing policies," Transp. Res. Rec. J. Transp. Res. Board, no. 1894, pp. 1-10, 2004.

[12] E. Jenelius, L.-G. Mattsson, and D. Levinson, "Traveler delay costs and value of time with trip chains, flexible activity scheduling and information," Transp. Res. Part B Methodol., vol. 45, no. 5, pp. 789-807, Jun. 2011.

[13] C.-H. Joh, H. Timmermans, and T. Arentze, "Measuring and predicting adaptation behavior in multidimensional activity-travel patterns," Transportmetrica, vol. 2, no. 2, pp. 153-173, 2006.

[14] K. Nagel, B. Kickhofer, and J. W. Joubert, "Heterogeneous tolls and values of time in multi-agent transport simulation," in ABMTRANS, pp. 762-768.

[15] M. Kouwenhoven et al., "New values of time and reliability in passenger transport in The Netherlands," Apprais. Transp., vol. 47, pp. 37-49, Nov. 2014.

[16] S. F. A. Baqueri, W. Ectors, M. S. Ali, L. Knapen, D. Janssens, and A.-U.-H. Yasar, "Estimation of Value of Time for a Congested Network - A Case Study of the National Highway, Karachi," 7th Int. Conf. Ambient Syst. Netw. Technol. ANT 2016 6th Int. Conf. Sustain. Energy Inf. Technol. SEIT-2016 Affil. Workshop, vol. 83, pp. 262-269, 2016.

[17] S. Hess, M. Bierlaire, and J. W. Polak, "Estimation of value of travel-time savings using mixed logit models," Posit. Util. Travel. Util. Travel, vol. 39, no. 2-3, pp. 221-236, Feb. 2005.

[18] C. Fezzi, I. J. Bateman, and S. Ferrini, "Using revealed preferences to estimate the Value of Travel Time to recreation sites," J. Environ. Econ. Manag., vol. 67, no. 1, pp. 58-70, Jan. 2014.

[19] D. Meunier and E. Quinet, "Value of Time Estimations in Cost Benefit Analysis: The French Experience," Curr. Pract. Transp. Apprais. Methods Policies Models - 42nd Eur. Transp. Conf. Sel. Proc., vol. 8, pp. 6271, Jan. 2015.

[20] T. Bellemans, B. Kochan, D. Janssens, G. Wets, T. Arentze, and H. Timmermans, "Implementation Framework and Development Trajectory of FEATHERS Activity-Based Simulation Platform," Transp. Res. Rec. J. Transp. Res. Board, no. Volume 2175 / 2010 Travel Forecasting 2010, Vol. 1, pp. 111-119, Dec. 2010.

[21] D. Ettema and H. Timmermans, "Modelling Departure Time Choice in the Context of Activity Scheduling Behavior," no. 1831, p. p 39-46, 2003.

[22] X. Zhang, H. Yang, H.-J. Huang, and H. M. Zhang, "Integrated scheduling of daily work activities and morning-evening commutes with bottleneck congestion," Transp. Res. Part Policy Pract., vol. 39, no. 1, pp. 41-60, Jan. 2005. 
[23] L. Knapen, T. Bellemans, M. Usman, D. Janssens, and G. Wets, "Within day rescheduling microsimulation combined with macrosimulated traffic," Adv. Comput. Commun. Their Impact Transp. Sci. Technol., vol. 45, pp. 99-118, Aug. 2014.

[24] K. Van Aerschot, "Analyse van het verband tussen de beperkte interesse in carpoolen en de inflexibiliteit van agenda's," Bachelor Verkeerskunde, Hasselt University, Diepenbeek, Belgium, 2014. 